\title{
Lyapunov Stability Analysis of a Delayed Foot-and-Mouth Disease Model with Animal Vaccination
}

\author{
Tinashe B. Gashirai, ${ }^{1}$ Senelani D. Hove-Musekwa, ${ }^{1}$ and Steady Mushayabasa $\mathbb{D}^{2}$ \\ ${ }^{1}$ Department of Applied Mathematics, National University of Science and Technology, P.O. Box 939, Ascot, Bulawayo, Zimbabwe \\ ${ }^{2}$ Department of Mathematics, University of Zimbabwe, P.O. Box MP 167, Harare, Zimbabwe \\ Correspondence should be addressed to Steady Mushayabasa; steadymushaya@gmail.com
}

Received 20 April 2020; Revised 8 June 2020; Accepted 10 June 2020; Published 5 August 2020

Academic Editor: Abdul Qadeer Khan

Copyright (c) 2020 Tinashe B. Gashirai et al. This is an open access article distributed under the Creative Commons Attribution License, which permits unrestricted use, distribution, and reproduction in any medium, provided the original work is properly cited.

\begin{abstract}
Foot-and-mouth disease virus remains one of the most important livestock diseases in sub-Saharan Africa and several Southeast Asian countries. Vaccination of livestock has been recognized as an important tool for the control of foot-and-mouth disease virus. However, this intervention strategy has some limitations. Generally, vaccine production is a complex multistep process which involves development, manufacturing, and delivery processes, and through this extensive process, some challenges such as poor vaccine storage often arise. More often, these challenges alter the validity of the vaccination. Foot-and-mouth disease virus epidemic dynamics have been extensively explored, but understanding the role of vaccination validity on virus endemicity is lacking. We present a time-delayed foot-and-mouth disease model that incorporates relevant biological and ecological factors, vaccination effects, and disease carriers. We determined the basic reproduction number and demonstrated that it is an important metric for persistence and extinction of the disease in the community. Numerical illustrations were utilised to support some of the analytical results.
\end{abstract}

\section{Introduction}

Foot-and-mouth disease (FMD) is one of the most important livestock diseases in sub-Saharan Africa and several Southeast Asian countries. It affects cloven-hoofed animals and is caused by an RNA virus commonly known as footand-mouth disease virus (FMDV). In many developed nations, the disease often occurs as epidemic (outbreak followed by extinction), whereas in many developing nations, such as Zimbabwe and Zambia and several Southeast Asian countries including Cambodia, Lao PDR, Malaysia, Myanmar, Thailand, and Vietnam, it is epidemic (persistent disease's presence) [1]. Epidemic or endemicity of the disease negatively affects agricultural economic world [2].

Among several other control strategies such as movement restrictions, public education, and culling, vaccination of animals has been recognized as the most important strategy to control the spread of the disease [3]. However, there are many limitations that affect vaccination validity period. For instance, cultivation of FMDV in the manufacturing units poses a risk of escape from production sites. Furthermore, vaccines may sometimes contain traces of FMD viral nonstructural proteins (NSPs), therefore, interfering with the NSP-based serological differentiation of infected from vaccinated animals [4]. In addition, vaccine production is a complex multistep process which involves development, manufacturing, and delivery processes, and through this extensive process, some challenges such as poor vaccine storage often arise. More often, these challenges alter the validity of the vaccination. For example, a study carried out in one Cambodian province recorded that more than half of cattle vaccinated with donated FMD vaccines are subsequently becoming infected with FMD virus and showing clinical signs of FMD indicating possible vaccine failure [1, 5]. Moreover, most vaccine production plants belong to companies, usually driven by profit rather than disease control or eradication; hence, most of the farmers in FMD endemic countries cannot afford the cost of these high- 
quality vaccines leading them to rely on donated or cheap vaccines obtained from unregistered suppliers, thereby compromising the outcome of vaccination programs $[6,7]$.

Since the 2001 FMD outbreak in UK, several mathematical models have been proposed to study the transmission and control of FMD (see, e.g., [6, 8-21]). Dexter [8] utilised stochastic epidemic models to explore the dynamics of FMD among feral pigs in the Australian semiarid rangelands. The author concluded that any control program designed to suppress FMD outbreak in feral pigs in the semiarid rangelands of Australia should take account of the prevailing environmental conditions. Using an SIR (susceptible-infectious-recovered) epidemic model, Bravo de Rueda et al. [9] demonstrated that both direct and indirect disease transmission pathways play a crucial role in FMD transmission. Utilising an nonautonomous mathematical model, Mushayabasa et al. [17] showed that seasonal variations may alter the dynamics of FMD. Recently, Zhang et al. [21] developed a dynamical switching model for FMD that incorporated three prevalent serotypes of the virus. The model was used to assess the spread of FMD in mainland China. Among several other important outcomes, their study showed that, under certain conditions, some of the FMD serotypes persist while others may disappear.

Despite all these efforts, however, mathematical models that aim to explore the role of vaccine waning in FMD endemic regions are still lacking. Therefore, in this study, we developed a time-delayed FMD model to explore the effects of vaccine waning on the dynamics of the disease. The proposed model incorporates all the relevant biological and ecological factors, vaccination, and disease carriers. We are aware that the role of FMD carriers in disease transmission remains a debatable issue $[17,22]$ and as such we have explored the outcomes of the model in two scenarios: (i) no transmission by carriers and (ii) when carriers are transmitting the disease. Time delay plays an important role in modeling the dynamics of infectious diseases, and the dynamical behaviour of an epidemic model with time delay(s) is known to be complex and difficult to comprehend [23]. In the proposed framework, the time delay represents the validity of the vaccination period.

We organize this paper as follows. In Section 2, we formulate and analyze the time-delayed FMD model. In particular, we computed the basic reproduction number and demonstrated that it is an important metric for the existence and global stability of the model steady states. In addition, we also explored the model numerically to support the analytical results. In the last section, we discuss the insightful implications of our findings on the role of vaccination validity on FMD transmission and control.

\section{Methods and Results}

In this section, we propose and analyze a time-delayed footand-mouth disease model incorporating direct and indirect disease transmission pathways, vaccination effects, and virus excreting carriers. We will investigate the qualitative behaviours of the proposed framework through studying the stability of the model steady states.
2.1. Model Framework. Let the variables $S(t), E(t), I(t)$, $A(t)$, and $R(t)$ represent the densities of susceptible, exposed (latently infected), clinical infectious, carrier, and recovered cattle at time $t$, respectively, such that the total population of cattle at any given time is $N(t)=S(t)+E(t)+I(t)+A(t)+R(t)$. In addition, let the variable $P(t)$ denote the density of the FMDV excreted into the environment by clinically infected and carrier animals. The proposed model is as follows:

$$
\left\{\begin{aligned}
S^{\prime}(t)= & \Pi-\beta_{1} I(t) S(t)-\beta_{1} \varepsilon A(t) S(t)-\beta_{2} P(t) S(t) \\
& -(\mu+p) S(t)+p e^{-\mu \tau} S(t-\tau) \\
E^{\prime}(t)= & \beta_{1} I(t) S(t)+\beta_{1} \varepsilon A(t) S(t)+\beta_{2} P(t) S(t)-(\mu+\gamma) E(t), \\
I^{\prime}(t)= & \gamma E(t)-(\mu+\phi) I(t), \\
A^{\prime}(t)= & f \phi I(t)-(\mu+\sigma) A(t), \\
P^{\prime}(t)= & \eta I(t)+\kappa \eta A(t)-\delta P(t)
\end{aligned}\right.
$$

$$
R^{\prime}(t)=(1-f) \phi I(t)+\sigma A(t)-\mu R(t),
$$

subject to the initial functions as follows:

$$
\left\{\begin{array}{l}
S(0)=\psi_{1}(\theta) \\
E(0)=\psi_{2}(\theta) \\
I(0)=\psi_{3}(\theta) \\
A(0)=\psi_{4}(\theta) \\
P(0)=\psi_{5}(\theta) \\
R(0)=\psi_{6}(\theta)
\end{array}\right.
$$

where

$$
\psi_{i}(\theta) \geq 0, \quad \theta \in[-\tau, 0], i=1,2,3,4,5,6,
$$

are continuous on $[-\tau, 0]$.

All model parameters are assumed to be positive and are defined as follows: $\Pi$ denotes the constant recruitment rate of animals through birth and they are assumed to be susceptible to infection. All animals are assumed to have a life span of $1 / \mu$ days, despite the epidemiological status of the animal. Susceptible animals are assumed to acquire infection either directly through contact with clinically infected animals or carriers at rate, $\beta_{1}$, or indirectly when they inhale the virus excreted into the environment by the aforementioned infectious animals; at rate $\beta_{2}, \varepsilon$ accounts for differential infectivity between clinically infected animals and carriers. Thus, $\varepsilon<1$ implies that clinically infected animals are assumed to be more infectious than carriers, and the reverse will be true, for $\varepsilon>1$. Model parameter $p$ is the proportion of vaccinated animals, the term $S(t-\tau) e^{-\mu \tau}$ represents the vaccinated animals, and $\tau$ is the validity period of the vaccination. Animals exposed to FMDV incubate the disease for a period ranging between 2 and 14 days [6, 16-18]. Here, $1 / \gamma$ represents the average incubation period.

FMD is rarely fatal and infected cattle generally clear the systemic infection within 8-15 days [22]. However, unlike pigs which usually clear FMDV within 3 weeks following infection and do not become carriers $[22,24-26]$, up to $50 \%$ 
of FMD-recovered ruminants become persistently infected [22]. Hence, we have assumed that, after an average infectious period of $1 / \phi$ days, a fraction $f$ of clinically infected animals becomes carriers and the remainder $(1-f)$ successfully clear the infection. Furthermore, compared to pigs, ruminants like cattle are highly susceptible to FMDV infection via the inhalation route $[22,27]$. Clinically infected and carrier animals shed the virus in the environment at rates $\eta$ and $\kappa \eta$, respectively, where $\kappa$ is a dimensionless parameter that accounts for the difference in pathogen shedding rate between animals from the aforementioned infectious classes. We assume that it takes $1 / \delta$ days for the pathogen in the environment to clear the infection. Furthermore, carriers are assumed to clear the infection after a duration of $1 / \sigma$ days. In this study, FMD carriers are animals from which the virus can be recovered more than 28 days after infection.

2.2. Properties of the Solutions. The following theorem shows that the model proposed in this study is biologically meaningful. Precisely, the theorem demonstrates that all the solutions of the proposed model are nonnegative and bounded for all $t>0$. Since the five equations of model (1) do not depend on (2), it suffices to study the dynamical behaviour of the disease without this equation.

Theorem 1. There exists a unique solution for the FMD model (1). Furthermore, the solution is nonnegative for all $t>0$ and lies in the set

$$
\begin{aligned}
\Omega=\{ & (S(t), E(t), I(t), A(t), P(t)) \in \mathbb{R}_{+}^{5}: S(t)+E(t)+I(t) \\
& \left.+A(t) \leq \frac{\Pi}{\mu}, P(t) \leq \frac{\eta \Pi}{\delta \mu}(\kappa+1)\right\} .
\end{aligned}
$$

Proof. In order to demonstrate that the solutions of model (1) are nonnegative, we will investigate the direction of the vector field given by the right-hand side of (1) on each space and note whether the vector field points to the interior of $\mathbb{R}_{+}^{5}$ or is tangent to the coordinate space. Since

$$
\left\{\begin{array}{l}
\left.S^{\prime}(t)\right|_{S=0}=\Pi \geq 0 \\
\left.E^{\prime}(t)\right|_{E=0}=\beta_{1} I(t) S(t)+\beta_{1} \varepsilon A(t) S(t)+\beta_{2} P(t) S(t) \geq 0 \\
\left.I^{\prime}(t)\right|_{I=0}=\gamma E(t) \geq 0 \\
\left.A^{\prime}(t)\right|_{A=0}=f \phi I(t) \geq 0 \\
\left.P^{\prime}(t)\right|_{P=0}=\eta I(t)+\kappa \eta A(t) \geq 0
\end{array}\right.
$$

it follows that the vector field given by the right-hand side of (1) on each coordinate plane either is tangent to the coordinate plane or points to the interior of $\mathbb{R}_{+}^{5}$. Hence, the domain $\mathbb{R}_{+}^{5}$ is a positively invariant region. Moreover, if the initial conditions $\psi_{i} \geq 0$ are nonnegative, then it follows that the corresponding solutions of model (1) are nonnegative.
Furthermore, let $M(t)=S(t)+E(t)+I(t)+A(t)$ and it follows that

$$
\begin{aligned}
M^{\prime}(t) & =S^{\prime}(t)+E^{\prime}(t)+I^{\prime}(t)+A^{\prime}(t) \\
& =\Pi-\mu M(t)-\phi I(t)-\sigma A(t) \\
& \leq \Pi-\mu M(t) .
\end{aligned}
$$

Since $M^{\prime}(t) \leq \Pi-\mu M(t)$, it follows by applying the standard comparison [28] that $M(t) \leq M(0) e^{-\mu t}+$ $(\Pi / \mu)\left(1-e^{-\mu t}\right)$. In particular, we $M(t) \leq \Pi / \mu$ if $M(0) \leq \Pi / \mu$. Hence we can conclude that the animal population is bounded. From the last equation of system (1),

$$
\begin{aligned}
P^{\prime}(t) & =\eta[I(t)+\kappa A(t)]-\delta P(t) \\
& \leq \frac{\eta(\kappa+1) \Pi}{\mu}-\delta P(t) .
\end{aligned}
$$

Similarly, by the standard comparison theorem [28], we have $P(t) \leq P(0) e^{-\delta t}+(\eta \Pi / \delta \mu)(\kappa+1)\left(1-e^{-\delta t}\right)$. Therefore, $P(t) \leq(\eta \Pi / \delta \mu)(\kappa+1)$, if $P(0) \leq(\eta \Pi / \delta \mu)(\kappa+1)$ and this implies that the pathogen population is also bounded. Hence, all solutions in $\mathbb{R}_{+}^{5}$ eventually enter $\Omega$.

2.3. Stability and Bifurcation Analysis. In this section, we investigate the qualitative behaviours of the delay differential model (1). In particular, we will determine the basic reproduction number, existence, and global stability of the model steady states. Through direct calculations, one can easily note that model (1) admits two equilibrium points, namely, the disease-free $\mathscr{E}^{0}$ and the endemic $\mathscr{E}^{*}$ defined by

$$
\begin{aligned}
\mathscr{E}^{0}:\left(S^{0}, E^{0}, I^{0}, A^{0}, P^{0}\right) & =\left(\frac{\mu}{\mu+p\left(1-e^{-\mu \tau}\right)}, 0,0,0,0\right), \\
\mathscr{E}^{*} & =\left(S^{*}, I^{*}, A^{*}, P^{*}\right),
\end{aligned}
$$

with

$$
\left\{\begin{array}{l}
S^{*}=\frac{\mu}{\left[\mu+p\left(1-e^{-\mu \tau}\right)\right] \mathscr{R}_{0}}, \\
E^{*}=\frac{\delta m_{2} m_{3}\left[\mu+p\left(1-e^{-\mu \tau}\right]\left[\mathscr{R}_{0}-1\right]\right.}{\beta_{1} \gamma \delta\left[f \phi \epsilon+m_{3}\right]+\beta \eta\left[\kappa f \phi+m_{3}\right]} \\
I^{*}=\frac{\delta m_{3}\left[\mu+p\left(1-e^{-\mu \tau}\right]\left[\mathscr{R}_{0}-1\right]\right.}{\beta_{1} \delta\left[f \phi \epsilon+m_{3}\right]+\beta \eta\left[\kappa f \phi+m_{3}\right]} \\
A^{*}=\frac{f \phi \delta\left[\mu+p\left(1-e^{-\mu \tau}\right]\left[\mathscr{R}_{0}-1\right]\right.}{\beta_{1} \delta\left[f \phi \epsilon+m_{3}\right]+\beta \eta\left[\kappa f \phi+m_{3}\right]} \\
P^{*}=\frac{\eta\left[\kappa f \phi+m_{3}\right]\left[\mu+p\left(1-e^{-\mu \tau}\right]\left[\mathscr{R}_{0}-1\right]\right.}{\beta_{1} \delta\left[f \phi \epsilon+m_{3}\right]+\beta \eta\left[\kappa f \phi+m_{3}\right]}
\end{array}\right.
$$

where $\mathscr{R}_{0}$, the basic reproduction number which is given by (1), is 


$$
\begin{aligned}
\mathscr{R}_{0} & =\mathscr{R}_{1}+\mathscr{R}_{2} \\
& =\frac{\beta_{1} \gamma\left(f \phi \epsilon+m_{3}\right) \mu}{\left(\mu+p\left(1-e^{-\mu \tau_{1}}\right) m_{1} m_{2} m_{3}\right.}+\frac{\beta_{2} \eta \gamma\left(\kappa f \phi+m_{3}\right) \mu}{\delta m_{1} m_{2} m_{3}\left(\mu+p\left(1-e^{-\mu \tau_{1}}\right)\right.},
\end{aligned}
$$

with

$$
\begin{aligned}
& m_{1}=(\mu+\gamma), \\
& m_{2}=(\mu+\phi), \\
& m_{3}=(\mu+\sigma) .
\end{aligned}
$$

The threshold quantity $\mathscr{R}_{0}$ is defined as the average number of secondary foot-and-mouth disease cases generated by a typical infected animal throughout its entire course of infection in a completely susceptible population in the presence of animal vaccination. The quantities $\mathscr{R}_{1}$ and $\mathscr{R}_{2}$ represent the basic reproduction number associated with direct and indirect disease transmission pathways, respectively. The basic reproduction number is an important metric for infectious disease models; it demonstrates the power of the disease to invade the population. From (17), we can observe that the disease persists if and only if $\mathscr{R}_{0}>1$ and it dies out for $\mathscr{R}_{0}<1$.

Next, we investigate the global stability of the diseasefree equilibrium, using a Lyapunov functional. We define a continuous and differentiable function $F: \mathbb{R}_{>0} \longrightarrow \mathbb{R}_{\geq 0}$ as

$$
F(z)=z-1-\ln z \text {. }
$$

We note that $F(z) \geq 0$ for any $z>0$ and has the global minimum 0 at $z=1$.

Theorem 2. Let $\psi \subset \Omega$ be a closed and bounded positively invariant set with respect to system (1) [29]. Let
$\mathscr{V}_{0}(t): \mathbb{R}_{+}^{5} \longrightarrow \mathbb{R}_{+}$be continuous and differentiable function such that $\mathscr{V}_{0}(t)>0$ and $\mathscr{V}_{0}^{\prime}(t) \leq 0$ in $\psi$ for $x(t)$ solutions of system (1). Let $E=\left\{x \in \Omega \mid \mathscr{V}_{0}^{\prime}(t)=0\right\}$. Let $\mathscr{M}$ be the largest invariant set in $E$; then every bounded solution starting in $\Omega$ approaches $\mathscr{M}$ as $t \longrightarrow \infty$. Particularly, when $\mathscr{M}=x^{*}=\mathscr{E}^{0}$, then $x \longrightarrow x^{*}$, as $t \longrightarrow \infty$.

Theorem 3. For system (1), when $\mathscr{R}_{0}<1$, the disease-free equilibrium point is globally asymptotically stable.

Proof. To establish the global stability of the DFE, we consider the following Lyapunov functional:

$$
\begin{aligned}
\mathscr{V}_{0}(t)= & a_{1}\left(S(t)-S^{0}-S^{0} \ln \frac{S(t)}{S^{0}}\right) \\
& +a_{1} p e^{-\mu \tau} \int_{t-\tau}^{t}\left(S(u)-S^{0}-S^{0} \ln \frac{S(u)}{S^{0}}\right) \mathrm{d} u \\
& +a_{1} E(t)+a_{2} I(t)+a_{3} A(t)+a_{4} P(t)
\end{aligned}
$$

where

$$
\begin{cases}a_{1}=\frac{\beta_{1} \gamma\left(f \phi \epsilon+m_{3}\right)}{m_{1} m_{2} m_{3}}+\frac{\beta_{2} \eta \gamma\left(\kappa f \phi+m_{3}\right)}{\delta m_{1} m_{2} m_{3}}, & a_{3}=\frac{\beta_{1} \epsilon \delta+\beta_{2} \kappa \eta}{\delta m_{3}}, \\ a_{2}=\frac{\beta_{1}\left(f \phi \epsilon+m_{3}\right)}{m_{2} m_{3}}+\frac{\beta_{2} \eta\left(\kappa f \phi+m_{3}\right)}{\delta m_{2} m_{3}}, & a_{4}=\frac{\beta_{2}}{\delta} .\end{cases}
$$

Taking the derivative of $\mathscr{V}_{0}(t)$ along the solutions of system (1) leads to

$$
\begin{aligned}
\mathscr{V}_{0}^{\prime}(t)= & a_{1}\left(1-\frac{S^{0}}{S(t)}\right) S^{\prime}(t)+a_{1} p e^{-\mu \tau}\left(S(t)-S(t-\tau)+S^{0} \ln \frac{S(t-\tau)}{S(t)}\right) \\
& +a_{1} E^{\prime}(t)+a_{2} I^{\prime}(t)+a_{3} A^{\prime}(t)+a_{4} P^{\prime}(t) \\
= & a_{1}\left(1-\frac{S^{0}}{S(t)}\right)\left[\Pi-\left(\beta_{1} I(t)+\beta_{1} \varepsilon A(t)+\beta_{2} P(t)\right) S(t)-(\mu+p) S(t)+a_{1} p e^{-\mu \tau} S(t-\tau)\right] \\
& +a_{1} p e^{-\mu \tau}\left(S(t)-S(t-\tau)+S^{0} \ln \frac{S(t-\tau)}{S(t)}\right) \\
& +a_{1}\left[\beta_{1} I(t) S(t)+\beta_{1} \in A(t) S(t)+\beta_{2} P(t) S(t)-m_{1} E(t)\right] \\
& +a_{2}\left[\gamma E(t)-m_{2} I(t)\right]+a_{3}\left[f \phi I(t)-m_{3} A(t)\right]+a_{4}[\eta I(t)+\kappa \eta A(t)-\delta P(t)] .
\end{aligned}
$$

At disease-free equilibrium, we have the identity $\Pi=(\mu+p) S^{0}-p e^{-\mu \tau} S^{0}$. Using this identity and simplifying, one obtains 


$$
\begin{aligned}
\mathscr{V}_{0}^{\prime}(t)= & \left(\mu+p\left(1-e^{-\mu \tau}\right)\right) S^{0}\left(\frac{\beta_{1} \gamma\left(f \phi \epsilon+m_{3}\right)}{m_{1} m_{2} m_{3}}+\frac{\beta_{2} \eta \gamma\left(\kappa f \phi+m_{3}\right)}{\delta m_{1} m_{2} m_{3}}\right)\left(2-\frac{S(t)}{S^{0}}-\frac{S^{0}}{S(t)}\right) \\
& +p e^{-\mu \tau} S^{0}\left(\frac{\beta_{1} \gamma\left(f \phi \epsilon+m_{3}\right)}{m_{1} m_{2} m_{3}}+\frac{\beta_{2} \eta \gamma\left(\kappa f \phi+m_{3}\right)}{\delta m_{1} m_{2} m_{3}}\right)\left(1-\frac{S(t-\tau)}{S(t)}+\ln \frac{S(t-\tau)}{S(t)}\right) \\
& +\left(\left[\frac{\beta_{1} \gamma\left(f \phi \epsilon+m_{3}\right)}{m_{1} m_{2} m_{3}}+\frac{\beta_{2} \eta \gamma\left(\kappa f \phi+m_{3}\right)}{\delta m_{1} m_{2} m_{3}}\right] S-1\right)\left(\beta_{1} I+\beta_{1} \epsilon A+\beta_{2} P\right) \\
& \leq\left(\left[\frac{\beta_{1} \gamma\left(f \phi \epsilon+m_{3}\right)}{m_{1} m_{2} m_{3}}+\frac{\beta_{2} \eta \gamma\left(\kappa f \phi+m_{3}\right)}{\delta m_{1} m_{2} m_{3}}\right] S^{0}-1\right)\left(\beta_{1} I+\beta_{1} \in A+\beta_{2} P\right) \\
& =\left(\mu+p\left(1-e^{-\mu \tau}\right)\right) S^{0}\left(\frac{\beta_{1} \gamma\left(f \phi \epsilon+m_{3}\right)}{m_{1} m_{2} m_{3}}+\frac{\beta_{2} \eta \gamma\left(\kappa f \phi+m_{3}\right)}{\delta m_{1} m_{2} m_{3}}\right)\left(2-\frac{S(t)}{S^{0}}-\frac{S^{0}}{S(t)}\right) \\
& +p e^{-\mu \tau} S^{0}\left(\frac{\beta_{1} \gamma\left(f \phi \epsilon+m_{3}\right)}{m_{1} m_{2} m_{3}}+\frac{\beta_{2} \eta \gamma\left(\kappa f \phi+m_{3}\right)}{\delta m_{1} m_{2} m_{3}}\right)\left(1-\frac{S(t-\tau)}{S(t)}+\ln \frac{S(t-\tau)}{S(t)}\right) \\
& +\left(\mathscr{R}_{0}-1\right)\left(\beta_{1} I+\beta_{1} \epsilon A+\beta_{2} P\right) .
\end{aligned}
$$

Since the arithmetic mean is greater than or equal to the geometric mean, we have

$$
\frac{S(t)}{S^{0}}+\frac{S^{0}}{S(t)} \geq 2 \sqrt{\frac{S(t)}{S^{0}} \cdot \frac{S^{0}}{S(t)}}=2,
$$

and it follows that

$$
\left(2-\frac{S(t)}{S^{0}}-\frac{S^{0}}{S(t)}\right) \leq 0
$$

Furthermore, we define a continuous and differentiable function $\Phi(x)=1-x+\ln x$, for $x>0$. It follows that $\Phi(x) \leq 0$, with the equality satisfied if and only if $x=1$. Thus, we can note that

$$
1-\frac{S(t-\tau)}{S(t)}+\ln \frac{S(t-\tau)}{S(t)}=\Phi\left(\frac{S(t-\tau)}{S(t)}\right)
$$

From the earlier discussion on initial conditions (3) as well as the properties of solutions of model system (1), we can have $S(0)=\psi_{1}(\theta) \geq 0$ for $\theta \in[-\tau, 0]$ and this implies that $S(t-\tau) \geq 0, \quad \forall t \geq 0$. Hence, it follows that $\Phi((S(t-\tau)) / S(t)) \leq 0$.

Hence, if $\mathscr{R}_{0} \leq 1$, we have $\mathscr{V}_{0}^{\prime}(t) \leq 0$. Furthermore, let $\mathscr{M}$ be the largest invariant subset of the set $\Omega=\left\{(S(t), E(t), I(t), A(t), P(t)) \mid \mathscr{V}_{0}^{\prime}(t)=0\right\}$. We now claim that $\mathscr{M}=\left\{\mathscr{C}^{0}\right\}$. In fact, when $\mathscr{R}_{0}<1$, it follows from (17) that $\Omega=\{(S(t), E(t), I(t), A(t), P$ $\left.(t)) \mid S(t)=S^{0}, E(t)=0, I(t)=0, A(t)=0, P(t)=0\right\}$, which leads to $\mathscr{M}=\left\{\mathscr{E}^{0}\right\}$. If $\mathscr{R}_{0}<1$ and
$\Omega=\left\{\left(S(t), E(t), I(t), A(t), P(t) \mid S(t)=S^{0}, E(t)=0, I(t)=\right.\right.$ $0, A(t)=0, P(t)=0\}$ from the first and second equations of system (1), we have $S(t)=S^{0}$. Again, we have $\mathscr{M}=\left\{\mathscr{E}^{0}\right\}$. Noting that $\mathscr{M}$ is invariant, by Lasalle's invariance principle [30], the disease-free equilibrium is globally asymptotically stable whenever $\mathscr{R}_{0} \leq 1$.

In what follows, we shall investigate the global stability of the endemic equilibrium $\mathscr{E}^{*}$ (we have shown that it exists whenever $\mathscr{R}_{0}>1$, i.e., $S^{*}>0, E^{*}, I^{*}, A^{*}>0$, and $P^{*}>0$ if and only if $\mathscr{R}_{0}>1$ ) by using the Lyapunov direct method. The construction of the Lyapunov functional is also based on the function $F(z)=z-1-\ln z$, by which we have already observed that it satisfies $F(z) \geq 0$ for any $z>0$ and has the global minimum 0 at $z=1$.

Theorem 4. Let $\psi_{1} \subset \Omega$ be a closed and bounded positively invariant set with respect to system (1) [29]. Let $\mathscr{V}_{1}(t): \mathbb{R}_{+}^{5} \longrightarrow \mathbb{R}_{+}$be continuous and differentiable function such that $\mathscr{V}_{1}(t)>0$ and $\mathscr{V}_{1}^{\prime}(t) \leq 0$ in $\psi_{1}$ for $x(t)$ solutions of system (1). Let $E^{*}=\left\{x \in \Omega \mid \mathscr{V}_{1}^{\prime}(t)=0\right\}$. Let $\mathscr{M}_{1}$ be the largest invariant set in $E^{*}$; then every bounded solution starting in $\Omega$ for $\mathscr{R}_{0}>1$ approaches $\mathscr{M}_{1}$ as $t \longrightarrow \infty$. Particularly, when $\mathscr{M}_{1}=x^{* *}=\mathscr{E}^{*}$, then $x \longrightarrow x^{* *}$, as $t \longrightarrow \infty$.

Theorem 5. If $\mathscr{R}_{0}>1$, then the endemic equilibrium of model system (1) exists and is globally asymptotically stable.

Proof. We consider a Lyapunov functional $\mathscr{V}_{1}(t)$ as follows:

$$
\begin{aligned}
\mathscr{V}_{1}(t)= & \left(S(t)-S^{*}-S^{*} \ln \frac{S(t)}{S^{*}}\right)+\left(E(t)-E^{*}-E^{*} \ln \frac{E(t)}{E^{*}}\right) \\
& +b_{1}\left(I(t)-I^{*}-I^{*} \ln \frac{I(t)}{I^{*}}\right)+b_{2}\left(A(t)-A^{*}-A^{*} \ln \frac{A(t)}{A^{*}}\right) \\
& +b_{3}\left(P(t)-P^{*}-P^{*} \ln \frac{P(t)}{P^{*}}\right)+p e^{-\mu \tau} \int_{t-\tau}^{t}\left(S(u)-S^{*}-S^{*} \ln \frac{S(u)}{S^{*}}\right) \mathrm{d} u,
\end{aligned}
$$


where

$$
\begin{aligned}
& b_{1}=\frac{g_{1}\left(I^{*}, A^{*}, P^{*}\right) S^{*}}{\gamma E^{*}}, \\
& b_{2}=\frac{g_{1}\left(I^{*}, A^{*}, P^{*}\right) S^{*}}{f \phi I^{*}}, \\
& b_{3}=\frac{g_{1}\left(I^{*}, A^{*}, P^{*}\right) S^{*}}{g_{2}\left(I^{*}, A^{*}\right)},
\end{aligned}
$$

with

$$
\begin{aligned}
g_{1}(I(t), A(t), P(t)) S(t)= & \beta_{1} I(t) S(t)+\beta_{1} \in A(t) S(t) \\
& +\beta_{2} P(t) S(t), \\
g_{2}(I(t), A(t))= & \eta I(t)+\kappa \eta A(t) .
\end{aligned}
$$

From Section 2.2, properties of solutions of model system (1), $\mathscr{V}_{1}(t)$ is well defined and $\mathscr{V}_{1}(t) \geq 0, \forall t \geq 0$. The equality holds if and only if $S(t)=S^{*}, E(t)=E^{*}, I(t)=I^{*}$, $A(t)=A^{*}$, and $P^{(t)}=P^{*}$.

Taking the derivative of $\mathscr{V}_{1}(t)$ along the solutions of system (1), one obtains

$$
\begin{aligned}
\mathscr{V}_{1}^{\prime}(t)= & \left(1-\frac{S^{*}}{S(t)}\right) S^{\prime}(t)+\left(1-\frac{E^{*}}{E(t)}\right) E^{\prime}(t) \\
& +b_{1}\left(1-\frac{I^{*}}{I(t)}\right) I^{\prime}(t)+b_{2}\left(1-\frac{A^{*}}{A(t)}\right) A^{\prime}(t) \\
& +b_{3}\left(1-\frac{P^{*}}{P(t)}\right) P^{\prime}(t) \\
& +p e^{-\mu \tau}\left(S(t)-S(t-\tau)+S^{*} \ln \frac{S(t-\tau)}{S(t)}\right) .
\end{aligned}
$$

At the endemic equilibrium of model system (1), we have the following identities:

$$
\left\{\begin{array}{l}
\Pi=g_{1}\left(I^{*}, A^{*}, P^{*}\right) S^{*}+\left(p\left(1-e^{-\mu \tau}+\mu\right) S^{*},\right. \\
m_{1} E^{*}=g_{1}\left(I^{*}, A^{*}, P^{*}\right) S^{*} \\
m_{2} I^{*}=\gamma E^{*} \\
m_{3} A^{*}=f \phi I^{*} \\
\delta P^{*}=g_{2}\left(I^{*}, A^{*}\right) .
\end{array}\right.
$$

Utilising the identities leads to

$$
\begin{aligned}
\mathscr{V}_{1}^{\prime}(t)= & \left(p\left(1-e^{-\mu \tau}\right)+\mu\right) S^{*}\left(2-\frac{S(t)}{S^{*}}-\frac{S^{*}}{S(t)}\right)+p e^{-\mu \tau} S^{*}\left(1-\frac{S(t-\tau)}{S(t)}+\ln \frac{S(t-\tau)}{S(t)}\right) \\
& +g_{1}\left(I^{*}, A^{*}, P^{*}\right) S^{*}\left(1-\frac{P}{P^{*}}-\frac{P^{*} g_{2}(I, A)}{P g_{2}\left(I^{*}, A^{*}\right)}+\frac{g_{2}(I, A)}{g_{2}\left(I^{*}, A^{*}\right)}\right) \\
& +g_{1}\left(I^{*}, A^{*}, P^{*}\right) S^{*}\left(4-\frac{S^{*}}{S}-\frac{S E^{*}}{S^{*} E} \frac{g_{1}(I, A, P)}{g_{1}\left(I^{*}, A^{*}, P^{*}\right)}+\frac{g_{1}(I, A, P)}{g_{1}\left(I^{*}, A^{*}, P^{*}\right)}-\frac{E I^{*}}{E^{*} I}-\frac{I A^{*}}{I^{*} A}-\frac{A}{A^{*}}\right) .
\end{aligned}
$$

Once again, since the arithmetic mean is greater than or equal to the geometric mean, we have

$$
\frac{S(t)}{S^{*}}+\frac{S^{*}}{S(t)} \geq 2 \sqrt{\frac{S(t)}{S^{*}} \cdot \frac{S^{*}}{S(t)}}=2,
$$

and it follows that

$$
\left(2-\frac{S(t)}{S^{*}}-\frac{S^{*}}{S(t)}\right) \leq 0
$$

Furthermore, as already illustrated on demonstrating the global stability of the disease-free equilibrium,

$$
1-\frac{S(t-\tau)}{S(t)}+\ln \frac{S(t-\tau)}{S(t)} \leq 0 .
$$
have

Moreover, using the function $\Phi(x)$, defined earlier, we 
TABle 1: Model parameters and their interpretations.

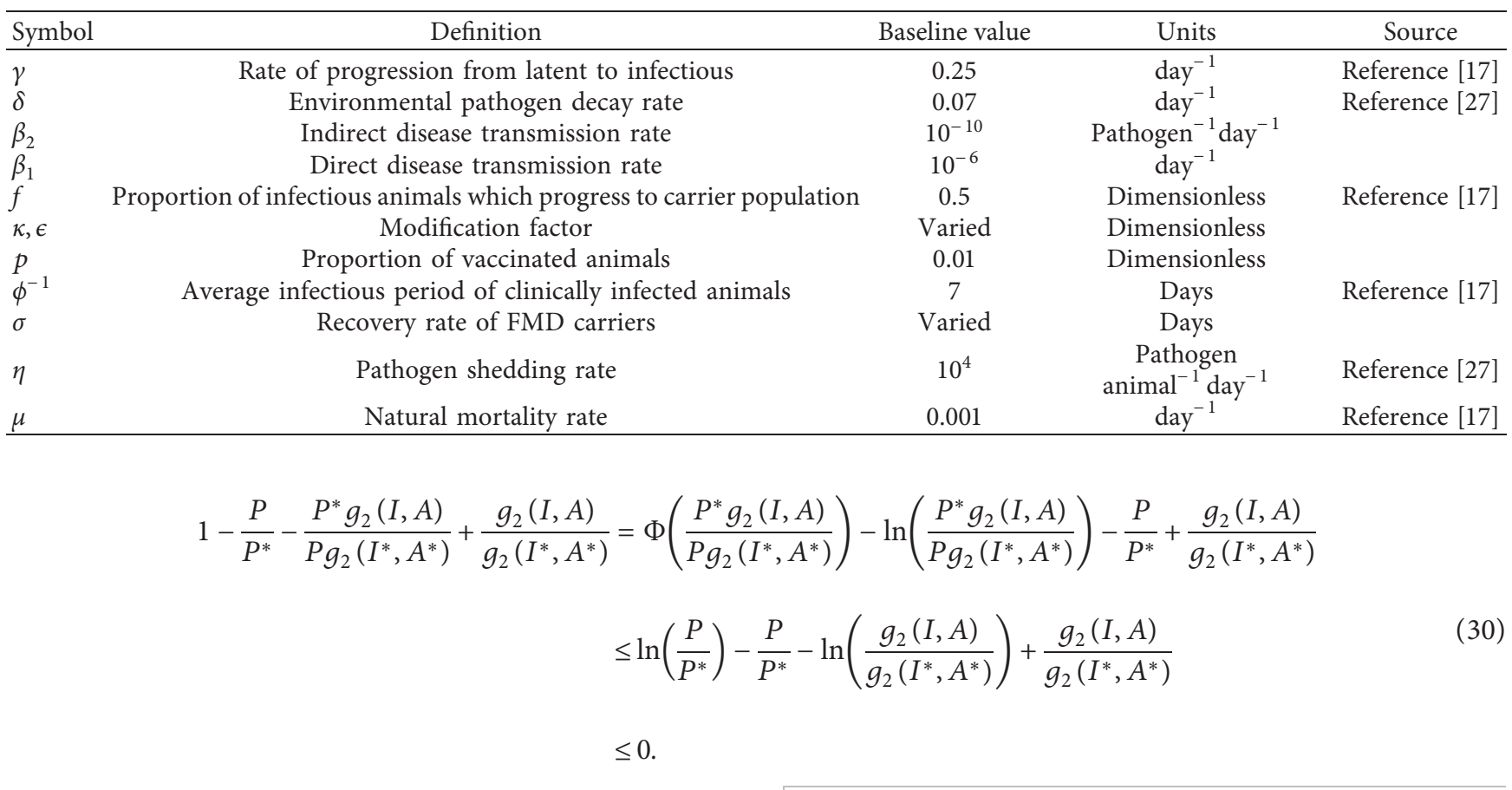

Furthermore,

$$
\begin{aligned}
4 & -\frac{S^{*}}{S}-\frac{S E^{*}}{S^{*} E} \frac{g_{1}(I, A, P)}{g_{1}\left(I^{*}, A^{*}, P^{*}\right)}+\frac{g_{1}(I, A, P)}{g_{1}\left(I^{*}, A^{*}, P^{*}\right)}-\frac{E I^{*}}{E^{*} I}-\frac{I A^{*}}{I^{*} A}-\frac{A}{A^{*}} \\
& =\Phi\left(\frac{S^{*}}{S}\right)+\Phi\left(\frac{E I^{*}}{E^{*} I}\right)+\Phi\left(\frac{I A^{*}}{I^{*} A}\right)+\Phi\left(\frac{S E^{*}}{S^{*} E} \frac{g_{1}(I, A, P)}{g_{1}\left(I^{*}, A^{*}, P^{*}\right)}\right) \\
& +\frac{g_{1}(I, A, P)}{g_{1}\left(I^{*}, A^{*}, P^{*}\right)}-\frac{A}{A^{*}}-\ln \left(\frac{A^{*} g_{1}(I, A, P)}{A g_{1}\left(I^{*}, A^{*}, P^{*}\right)}\right) \\
& \leq \ln \left(\frac{A}{A^{*}}\right)-\frac{A}{A^{*}}+\frac{g_{1}(I, A, P)}{g_{1}\left(I^{*}, A^{*}, P^{*}\right)}-\ln \left(\frac{g_{1}(I, A, P)}{g_{1}\left(I^{*}, A^{*}, P^{*}\right)}\right) \\
& \leq 0 .
\end{aligned}
$$

Hence, from the illustrations (28)-(31), if $\mathscr{R}_{0}>1$, we have $\mathscr{V}_{1}^{\prime}(t) \leq 0$. By Theorem 5.3.1 in [31], solutions are limited to $\mathscr{M}$, the largest invariant subset of $\left\{\mathscr{V}_{1}^{\prime}(t)=0\right\}$. Therefore, for all $(S(t), E(t), I(t), A(t), P(t)) \geq 0$ provided that $S^{*}, E^{*}, I^{*}, A^{*}, P^{*}$ are nonnegative, it follows by Lasalle's invariance principle [30] that the endemic equilibrium point is globally asymptotically stable whenever $\mathscr{R}_{0}>1$.

2.4. Numerical Results. To explore the behaviour of system (1) and illustrate the stability of equilibria solutions, we utilised dde23 [32] based on Runge-Kutta methods. The baseline values for the model parameters are in Table 1, and the initial population levels were assumed as follows: $S(0)=10^{4}$, $E(0)=200, I(0)=50, A(0)=0$, and $P(0)=10^{3}$.

Simulation results in Figures 1-3 illustrate the dynamical solutions of model (1) under the assumption that FMD carriers are noninfectious; that is, $\kappa=\epsilon=0$. In all the cases, we can note the existence of periodic oscillations for a certain period, before stability at endemic point is attained. We can also note that the intensity and amplitude of oscillations decrease as the vaccination validity period increases, implying that if the vaccine waning period is "reasonably longer," then the possibility of having periodic oscillations on the solutions of system (1) decreases. 

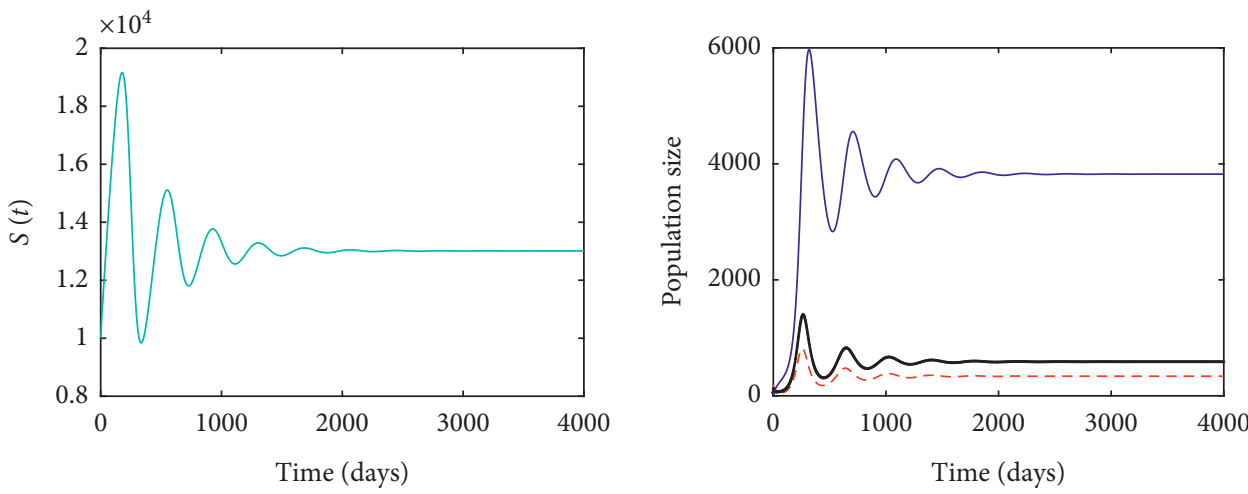

$$
\begin{aligned}
& --E(t) \\
& -I(t) \\
& -A(t)
\end{aligned}
$$

(a)

(b)

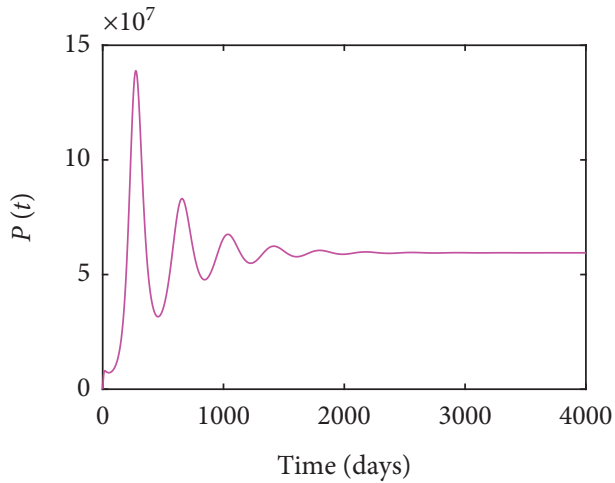

(c)



(d)

FIgURE 1: Numerical results of system (1) depicting the persistence of the disease of $\mathscr{R}_{0}>1$ when the vaccination validity $\tau$ is 15 days. Parameter values used to construct the simulations are in Table 1, with $p=0.01$ and $\kappa=\epsilon=0$, leading to $\mathscr{R}_{0}=6.7$.
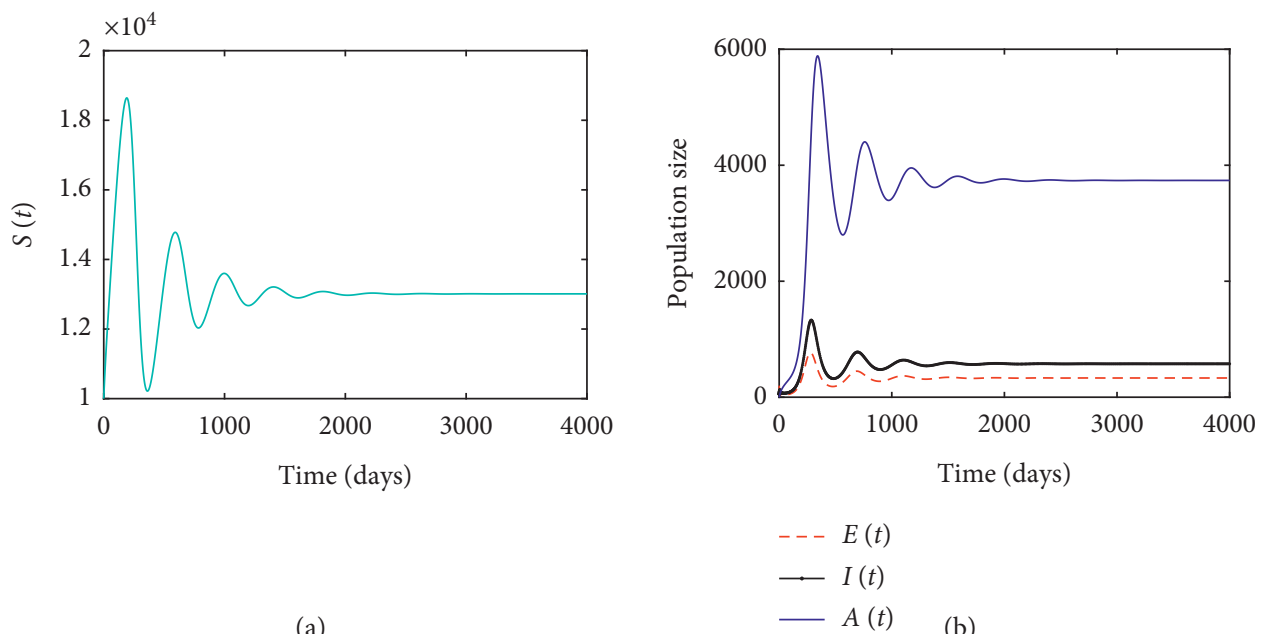

(a)

Figure 2: Continued. 


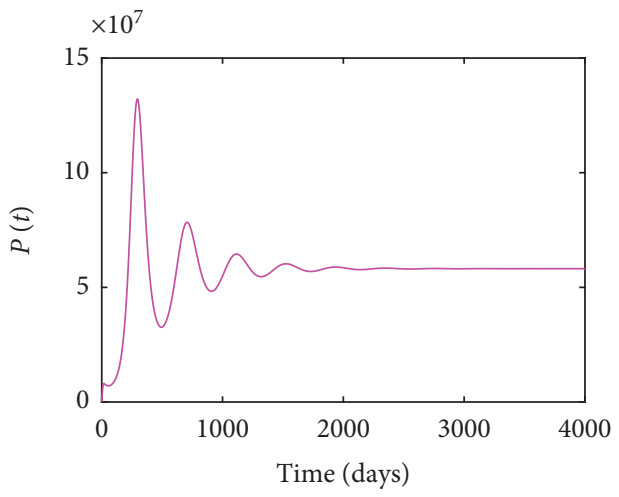

(c)



(d)

FIgURE 2: Numerical results of system (1) depicting the persistence of the disease of $\mathscr{R}_{0}>1$ when the vaccination validity $\tau$ is 30 days. Parameter values used to construct the simulations are in Table 1 , with $p=0.01$ and $\kappa=\epsilon=0$, leading to $\mathscr{R}_{0}=5.9$.
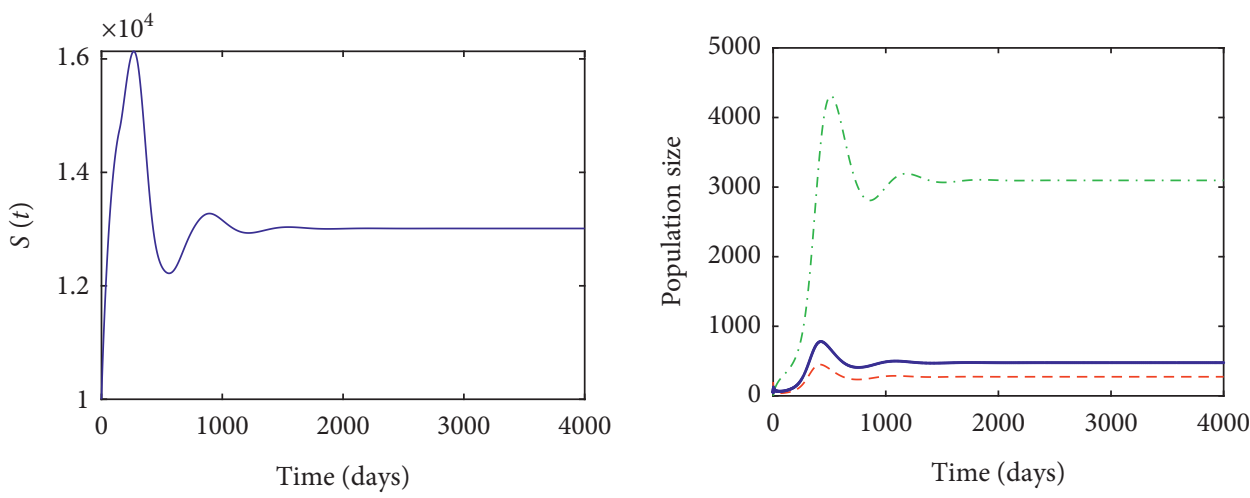

$$
\begin{aligned}
& --E(t) \\
& -I(t) \\
& -.-A(t)
\end{aligned}
$$

(a)

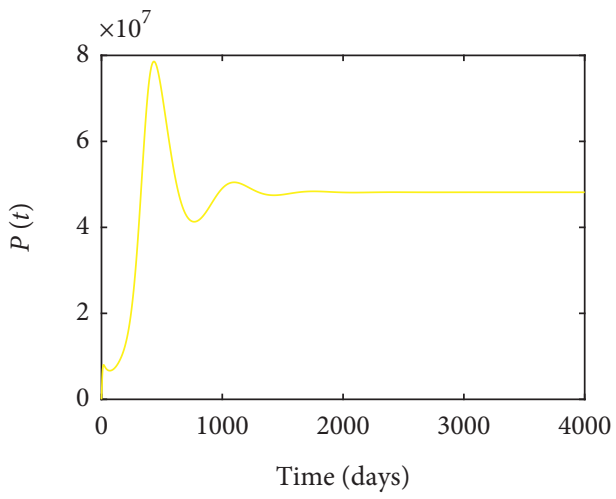

(c)

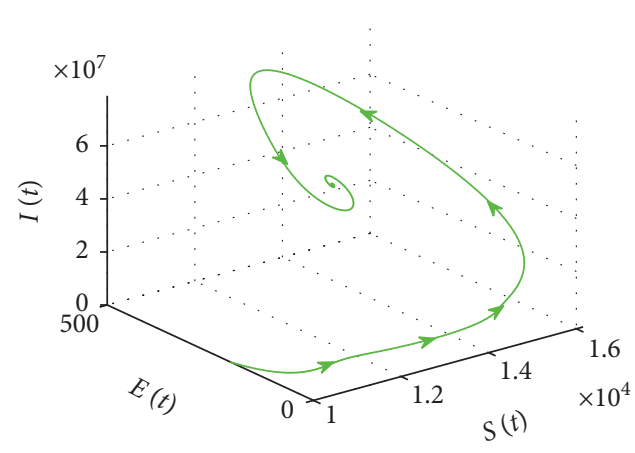

(d)

FIgURE 3: Numerical results of system (1) depicting the persistence of the disease of $\mathscr{R}_{0}>1$ when the vaccination validity $\tau$ is 150 days. Parameter values used to construct the simulations are in Table 1 , with $p=0.01$ and $\kappa=\epsilon=0$, leading to $\mathscr{R}_{0}=3.2$. 

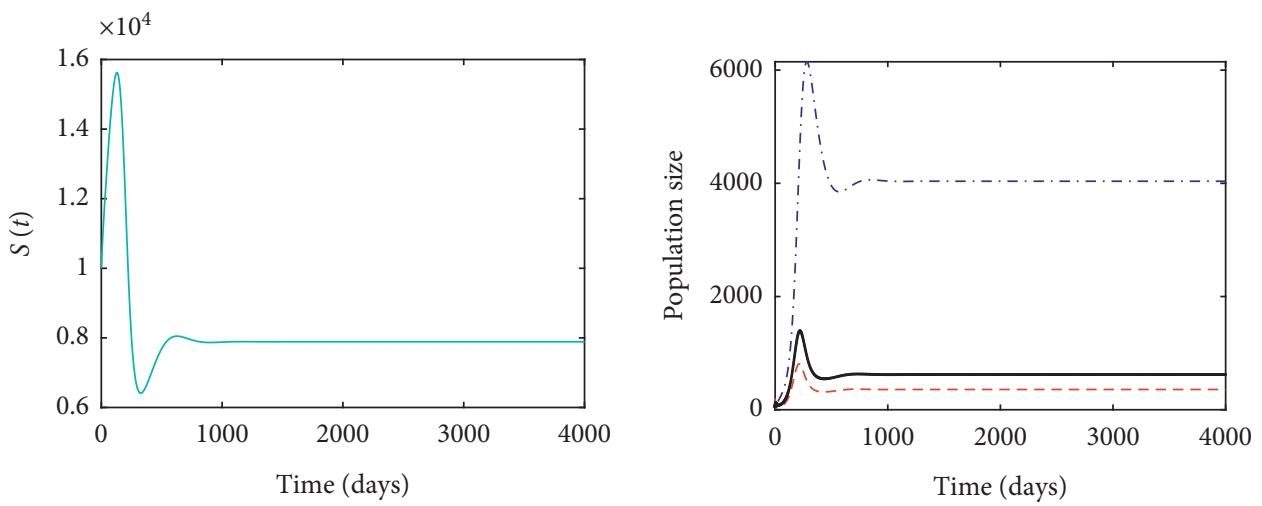

$$
\begin{aligned}
& --E(t) \\
& -\quad I(t) \\
& -\cdots A(t)
\end{aligned}
$$

(a)

(b)



(c)

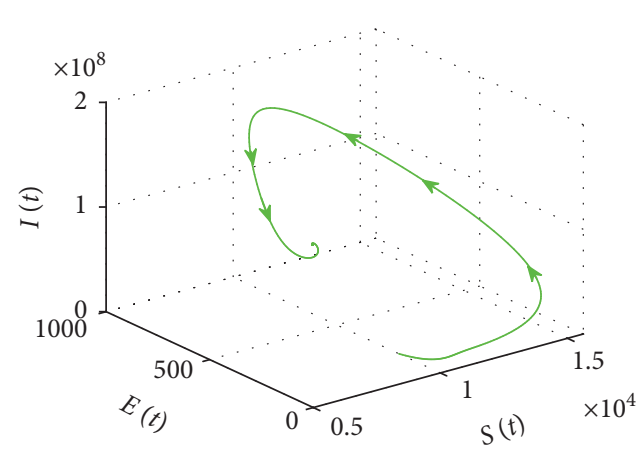

(d)

FIgURE 4: Numerical results of system (1) depicting the persistence of the disease of $\mathscr{R}_{0}>1$ when the vaccination validity $\tau$ is 30 days. Parameter values used to construct the simulations are in Table 1 , with $f=0.5, p=0.01$, and $\kappa=\epsilon=0.1$, leading to $\mathscr{R}_{0}=9.8$.
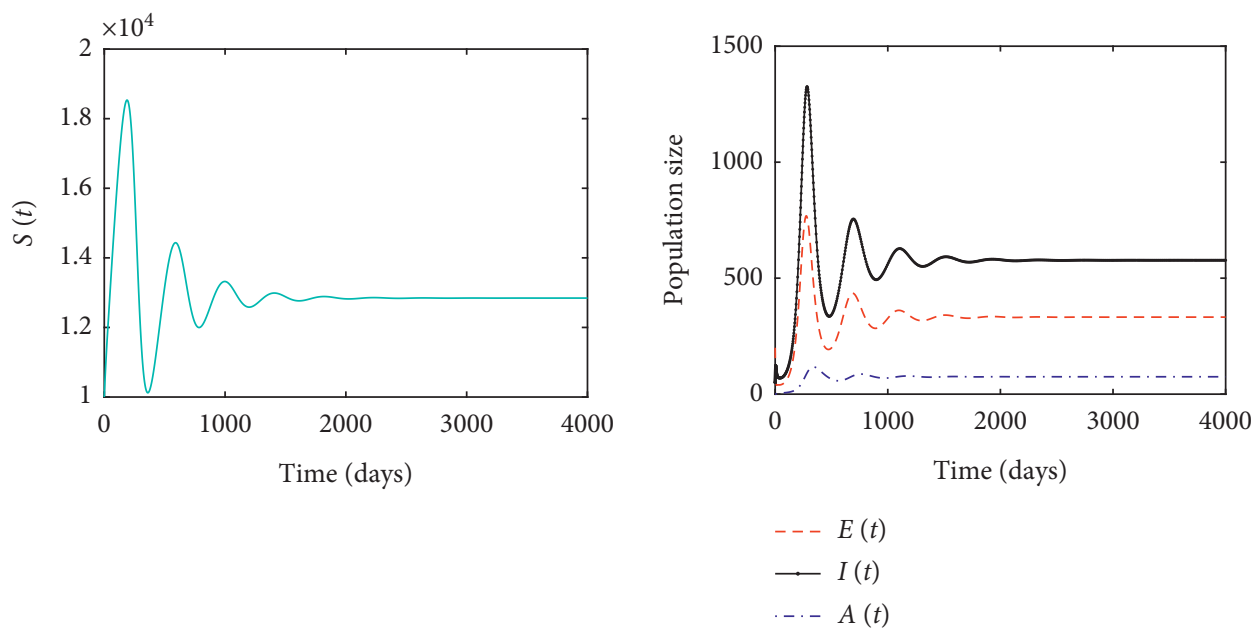

(a)

(b)

Figure 5: Continued. 




(c)

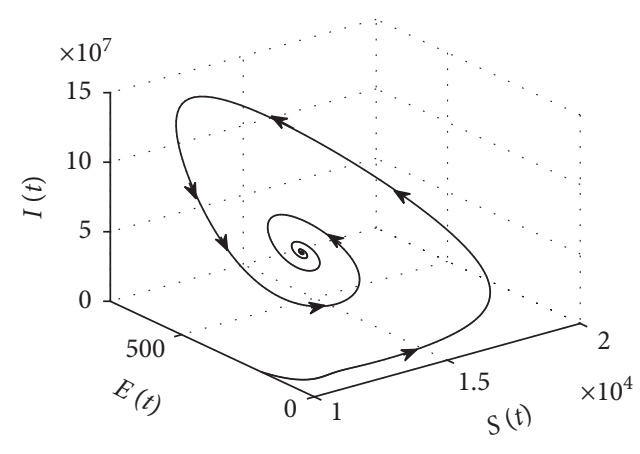

(d)

Figure 5: Numerical results of system (1) depicting the persistence of the disease of $\mathscr{R}_{0}>1$ when the vaccination validity $\tau$ is 30 days. Parameter values used to construct the simulations are in Table 1 , with $p=0.01, \kappa=\epsilon=0.1$, and $f=0.01$, leading to $\mathscr{R}_{0}=6.0$.
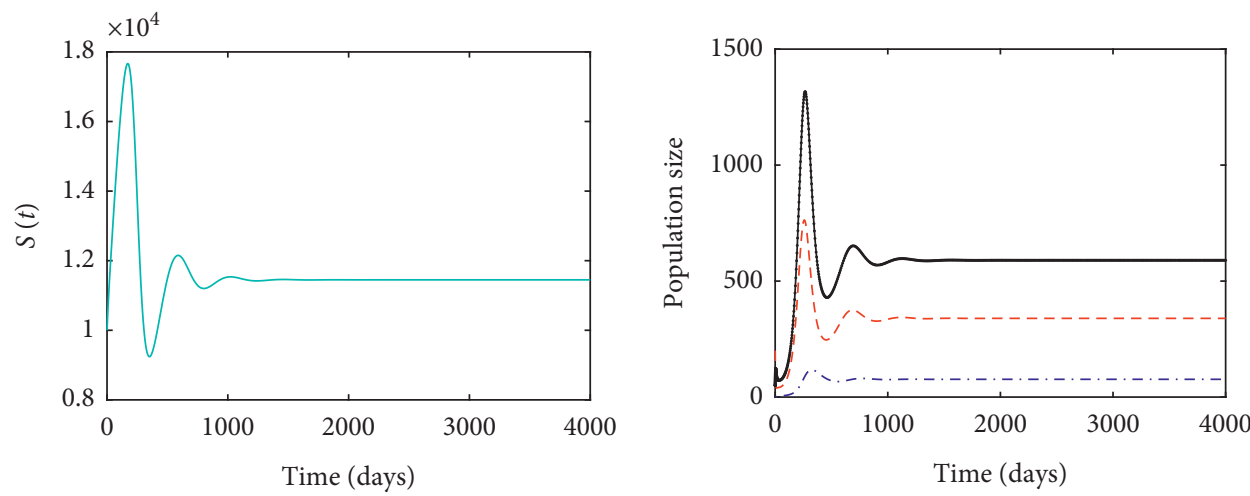

$$
\begin{aligned}
& --E(t) \\
& -\quad I(t) \\
& -.-A(t)
\end{aligned}
$$

(a)

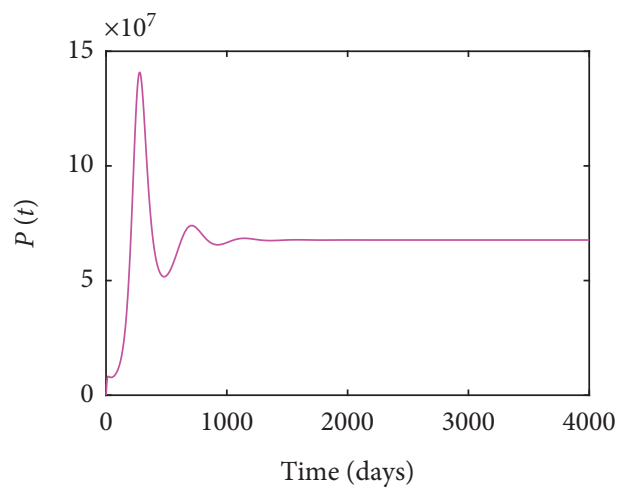

(c)

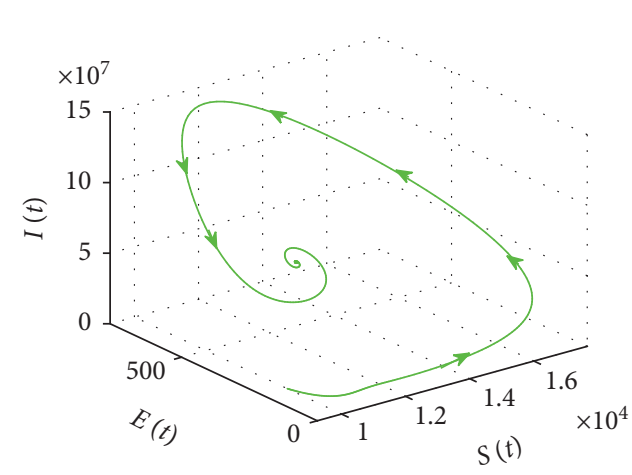

(d)

FIgURE 6: Numerical results of system (1) depicting the persistence of the disease of $\mathscr{R}_{0}>1$ when the vaccination validity $\tau$ is 30 days. Parameter values used to construct the simulations are in Table 1 , with $p=0.01, \kappa=\epsilon=1.05$, and $f=0.01$, leading to $\mathscr{R}_{0}=6.7$.

Graphical results in Figures 4-6 demonstrate the potential impact of infecting FMD carriers on altering the dynamics of foot-and-mouth disease. The contribution of FMD carriers on the disease dynamics remains a debatable issue. Some researchers argue that these animals are noninfectious, while others postulate that they are infectious [22]. However, a recent study [22] demonstrated that FMD carrier cattle are capable of shedding the virus into the environment. Thus, in Figures 4-6, we fixed the vaccination validity period to 30 days and explored the role of 
FMD carrier cattle on the dynamics of the disease by varying model parameters associated with increasing or decreasing the infectivity of the FMD carriers. In Figure 4, we set $f=0.5, p=0.01$, and $\kappa=\epsilon=0.1$, leading to $\mathscr{R}_{0}=9.8$. We observed that if the proportion of animals which become carriers, $f$, is large while the contribution of carriers to new infections (through transmission, $\epsilon$, and pathogen shedding into the environment, $\kappa$ ) is less than that of clinically infected animals, then intensity and amplitude of periodic oscillation in the solutions of model system (1) decrease. However, results in Figures 5 and 6 suggest that if the proportion of animals that become carriers decreases, then the intensity and amplitude of periodic solutions increase despite irrespective of whether carriers are less or more infectious compared to clinically infected animals. Overall, the results demonstrate that understanding the contribution of carriers on FMD dynamics is an important issue that requires multidimensional research.

\section{Concluding Remarks}

Foot-and-mouth disease, a highly contagious, acute viral disease of cloven-hoofed domestic and wild ruminants including cattle, buffalo, swine, goats, and sheep, remains a major challenge in many developing nations in the subSahara Africa, such as Zimbabwe and Zambia. Vaccination is regarded as the most effective means of foot-and-mouth disease mitigation. Nevertheless, this important intervention strategy has some limitations. Generally, vaccine production is a complex multistep process which involves development, manufacturing, and delivery processes, and through this extensive process, challenges such as poor vaccine storage often arise. Such challenges are known to affect the validity period of the vaccines. In this work, we proposed and analyzed a time-delayed foot-and-mouth disease model that incorporates vaccine waning and foot-and-mouth disease carriers. We are aware that the role of FMD carriers in disease transmission remains a debatable issue $[17,22]$ and as such we have explored the outcomes of the model in two scenarios: (i) no transmission by carriers and (ii) when carriers are transmitting the disease. We computed the basic reproduction number and demonstrated that it is an important threshold parameter for the existence and global stability of the model steady states. Utilising numerical illustrations, we demonstrated the impact of foot-and-mouth disease carriers on long- and short-term dynamics of the disease.

This work demonstrated that understanding the role of vaccine waning and FMD carriers is an important topic that requires multidimensional research. Nevertheless, this work is not exhaustive; it can be extended by adding diffusion terms to the proposed model so as to represent the movement and dispersal of the animals and the pathogen and by adding convection terms to represent the migration of animals.

\section{Data Availability}

The data used to support the findings of this study are included within the article.

\section{Conflicts of Interest}

The authors declare that they have no conflicts of interest.

\section{References}

[1] S. Sieng and J. Kerr, "Investigation of vaccination effectiveness in two Cambodian villages facing an outbreak of foot-andmouth disease," in Cattle: Cattle Health, Production and Trade in Cambodia, J. R. Young, L. Rast, S. Suon, and P. A. Windsor, Eds., pp. 67-71, ACIAR Proceedings No.138 Australian Centre for International Agricultural Research, Canberra, Australia, 2013.

[2] P. M. Schnell, Y. Shao, L. W. Pomeroy, J. H. Tien, M. Moritz, and R. Garabed, "Modeling the role of carrier and mobile hers on foot-and-mouth disease virus endemicty in the far north region of Cameroon," Epidemics, vol. 29, Article ID 100355, 2019.

[3] C. Bravo de Rueda, A. Dekker, P. L. Eblé, and M. C. M. De Jong, "Vaccination of cattle only is sufficient to stop FMDV transmission in mixed populations of sheep and cattle," Epidemiology and Infection, vol. 143, no. 11, pp. 2279-2286, 2015.

[4] Y. Cao, Z. Lu, and Z. Liu, "Foot-and-mouth disease: progress and problems," Expert Review of Vaccines, vol. 15, no. 6, 2016.

[5] S. Sieng, S. W. Walkden-Brown, and J. Kerr, "Effect of vaccine storage temperatures and dose rate on antibody responses to foot and mouth disease vaccination in Cambodia," Veterinary Medicine and Science, vol. 4, no. 1, pp. 35-44, 2018.

[6] T. B. Gashirai, S. D. Musekwa-Hove, P. O. Lolika, and S. Mushayabasa, "Global stability and optimal control analysis of a foot-and-mouth disease model with vaccine failure and environmental transmission," Chaos, Solitons \& Fractals, vol. 132, Article ID 109568, 2020.

[7] M. Rweyemamu, F. Maree, C. Kasanga et al., "Challenges and prospects for the control of foot-and-mouth disease: an African perspective," Veterinary Medicine: Research and Reports, vol. 5, pp. 119-138, 2014.

[8] N. Dexter, "Stochastic models of foot and mouth disease in feral pigs in the Australian semi-arid rangelands," Journal of Applied Ecology, vol. 40, no. 2, pp. 293-306, 2003.

[9] C. Bravo de Rueda, M. C. de Jong, P. L. Eblé, and A. Dekker, "Quantification of transmission of foot-and-mouth disease virus caused by an environment contaminated with secretions and excretions from infected calves," Veterinary Research, vol. 46, no. 1, p. 43, 2015.

[10] M. E. Chase-Topping, I. Handel, B. M. Bankowski et al., "Understanding foot-and-mouth disease virus transmission biology: identification of the indicators of infectiousness," Veterinary Research, vol. 44, no. 1, p. 46, 2013.

[11] D. M. Green, I. Z. Kiss, and R. R. Kao, "Modelling the initial spread of foot-and-mouth disease through animal movements," Proceedings of the Royal Society B: Biological Sciences, vol. 273, no. 1602, pp. 2729-2735, 2006.

[12] R. R. Kao, L. Danon, D. M. Green, and I. Z. Kiss, "Demographic structure and pathogen dynamics on the network of livestock movements in Great Britain," Proceedings of the Royal Society B: Biological Sciences, vol. 273, no. 1597, pp. 1999-2007, 2006. 
[13] M. J. Keeling, M. E. J. Woolhouse, R. M. May, G. Davies, and B. T. Grenfell, "Modelling vaccination strategies against footand-mouth disease," Nature, vol. 421, no. 6919, pp. 136-142, 2003.

[14] R. P. Kitching, "Identification of foot and mouth disease virus carrier and subclinically infected animals and differentiation from vaccinated animals," Revue Scientifique et Technique de l'OIE, vol. 21, no. 3, pp. 531-538, 2002.

[15] R. P. Kitching, A. M. Hutber, and M. V. Thrusfield, “A review of foot-and-mouth disease with special consideration for the clinical and epidemiological factors relevant to predictive modelling of the disease," The Veterinary Journal, vol. 169, no. 2, pp. 197-209, 2005.

[16] S. Mushayabasa, C. P. Bhunu, and M. Dhlamini, "Impact of vaccination and culling on controlling foot and mouth disease: a mathematical modelling approach," World Journal of Vaccines, vol. 1, no. 4, pp. 156-161, 2011.

[17] S. Mushayabasa, D. Psosny, and J. Wang, "Modeling the intrinsic dynamics of FMD," Molecular Biology and Evolution, vol. 13, pp. 425-442, 2015.

[18] S. Mushayabasa and G. Tapedzesa, "Modeling the effects of multiple intervention strategies on controlling foot-andmouth disease," BioMed Research International, vol. 2015, Article ID 584234, 10 pages, 2015

[19] N. Ringa and C. T. Bauch, "Impacts of constrained culling and vaccination on control of foot and mouth disease in nearendemic settings: a pair approximation model," Epidemics, vol. 9, pp. 18-30, 2014.

[20] G. Rossi, R. Smith, S. Pongolini, and L. Bolzoni, "Modelling farm-to-farm disease transmission through personnel movements: from visits to contacts, and back," Scientific Reports, vol. 7, no. 1, p. 2375, 2017.

[21] J. Zhang, Z. Jin, and Y. Yuan, "Assessing the spread of foot and mouth disease in mainland China by dynamical switching model," Journal of Theoretical Biology, vol. 460, pp. 209-219, 2019.

[22] A. B. R. Parthiban, M. Mahapatra, S. Gubbins, and S. Parida, "Virus excretion from foot-and- mouth disease virus carrier cattle and their potential role in causing new outbreaks," PLoS One, vol. 10, no. 6, Article ID e0128815, 2015.

[23] J. K. Hale and W. Z. Huang, "Global geometry of the stable regions for two delay differential equations," Journal of Mathematical Analysis and Applications, vol. 178, no. 2, pp. 344-362, 1993.

[24] S. Alexandersen, M. Quan, C. Murphy, J. Knight, and Z. Zhang, "Studies of quantitative parameters of virus excretion and transmission in pigs and cattle experimentally infected with foot-and-mouth disease virus," Journal of Comparative Pathology, vol. 129, no. 4, pp. 268-282, 2003.

[25] S. Parida, L. Fleming, Y. Oh et al., "Reduction of foot-andmouth disease (FMD) virus load in nasal excretions, saliva and exhaled air of vaccinated pigs following direct contact challenge," Vaccine, vol. 25, no. 45, pp. 7806-7817, 2007.

[26] C. Stenfeldt, J. M. Pacheco, L. L. Rodriguez, and J. Arzt, "Early events in the pathogenesis of foot-and-mouth disease in pigs; identification of oropharyngeal tonsils as sites of primary and sustained viral replication," PLoS One, vol. 9, no. 9, Article ID e106859, 2014.

[27] J. Slingluff, "Risk assessment for the transmission of foot-andmouth disease via movement of swine and cattle carcases from infected FMD-infected premises to a disposal site," 2014, https://conservancy.umn.edu/handle/11299/193839.
[28] V. Lakshmikantham, S. Leela, and A. A. Martynyuk, Stability Analysis of Nonlinear Systems, Marcel Dekker, New York, NY, USA, 1989.

[29] J. Huo, H. Zhao, and L. Zhu, "The effect of vaccines on backward bifurcation in a fractional order HIV model," Nonlinear Analysis: Real World Applications, vol. 26, pp. 289-305, 2015.

[30] J. S. LaSalle, "The stability of dynamical systems," in CBMSNSF Regional Conference Series in Applied Mathematics, Vol. 25, SIAM, Philadelphia, PA, USA, 1976.

[31] J. K. Hale and S. Verduyn Lunel, Introduction to Functional Differential Equations, Springer, New York, NY, USA, 1993.

[32] L. F. Shampine and S. Thompson, "Solving DDEs in MATLAB," Applied Numerical Mathematics, vol. 37, no. 4, pp. 441-458, 2001. 